\title{
Review Article \\ Pressure Sensor: State of the Art, Design, and Application for Robotic Hand
}

\author{
Ahmed M. Almassri, ${ }^{1}$ W. Z. Wan Hasan, ${ }^{1}$ S. A. Ahmad, ${ }^{1}$ A. J. Ishak, ${ }^{1}$ A. M. Ghazali, \\ D. N. Talib, ${ }^{1}$ and Chikamune Wada ${ }^{2}$ \\ ${ }^{1}$ Department of Electrical and Electronic Engineering, Faculty of Engineering, Universiti Putra Malaysia (UPM), 43400 Serdang, \\ Selangor, Malaysia \\ ${ }^{2}$ Graduate School of Life Science and Systems Engineering, Kyushu Institute of Technology, 2-4 Hibikino, Wakamatsu-ku, \\ Kitakyushu 808-0196, Japan
}

Correspondence should be addressed to Ahmed M. Almassri; eng.ahmed8989@gmail.com and W. Z. Wan Hasan; wanzuha@upm.edu.my

Received 5 November 2014; Accepted 15 December 2014

Academic Editor: Guangming Song

Copyright (C) 2015 Ahmed M. Almassri et al. This is an open access article distributed under the Creative Commons Attribution License, which permits unrestricted use, distribution, and reproduction in any medium, provided the original work is properly cited.

\begin{abstract}
We survey the state of the art in a variety of force sensors for designing and application of robotic hand. Most of the force sensors are examined based on tactile sensing. For a decade, many papers have widely discussed various sensor technologies and transducer methods which are based on microelectromechanical system (MEMS) and silicon used for improving the accuracy and performance measurement of tactile sensing capabilities especially for robotic hand applications. We found that transducers and materials such as piezoresistive and polymer, respectively, are used in order to improve the sensing sensitivity for grasping mechanisms in future. This predicted growth in such applications will explode into high risk tasks which requires very precise purposes. It shows considerable potential and significant levels of research attention.
\end{abstract}

\section{Introduction}

Robotic hand is a mechatronic machine that is made to complete assignment whenever it is required, especially for repetitive and dangerous tasks, and also during specific applications such as military robots, home automation [1], automotive industries, and nuclear industry robots. In fact, many robotic hand applications were already developed as in $[2,3]$; for instance, dexterous manipulation [4-6], tactile image perception [7, 8], artificial limbs [9], fingerprint recognition [10], grasping objects [11-13], and pick and place applications $[14,15]$ can also be widely seen in various industries. Nonetheless, some of these robotic applications require a lot of labor force, notably in terms of assembly line and material handling.

Henceforth, there is a significant need to have a dedicated machine, which is suitable for robust robotic application. For example, robotic hands manufacturing industries for pick and place mechanism are programed to complete task where it takes a product from one spot and put it to a different location. This technology has the advantages of reducing the risk process associated with human operators during the manufacturing process. Besides, it also saves time and energy required for the labor. Therefore, tactile sensing in the robotic hand is defined as a sensor device that is good enough to measure various properties of an object and provide information through physical touch between a sensor and an object [16]. Recently, the enhancement of the robotic hand sensor has received a substantial attention and becomes crucial to our everyday life. Researchers have recognized that equipping a robot with different sensors is a way to perform tasks in unstructured environment and enable the robot to cope with significant uncertainties. Due to the demand of ensuring safety between robots and objects during the mechanical touch, intelligent tactile sensing in robotic hand with high capabilities is critical.

In this paper, the different techniques for measuring force or interface pressures are presented. These techniques include 
load cells, pressure indicating film, and tactile pressure system. Similarly, a review on industry pressure sensing that involves the pick and place applications and algorithm control is also highlighted. The paper also discusses the MEMS sensor technology and different types of sensors while the last section of this part discusses the piezoresistive flexiForce sensor. FlexiForce sensor has a good substrate material, which is a polymer that enhances the force sensing and improves the performance of force, linearity, hysteresis, drift, and temperature sensitivity compared to any other thin film. Furthermore, it is flexible and ultrathin enough as the researchers and designers can use it in different integrated applications as well as for applications that are oriented to manipulative tasks with grippers of robotic hand. In a nutshell, new applications for tactile pressure sensing show a high increase in publications and research attention as viewed in Table 2. As a result, the design of sensor becomes more precise with higher reliability to overcome the problems.

\section{Sense of Pressure: Methods}

Pressure is force per unit area applied in a direction perpendicular to the surface of an object. The formula is commonly written as follows:

$$
P=\frac{F}{A},
$$

where $P$ is the pressure, $F$ is the normal force, and $A$ is the area of the surface of contact. When two objects are contacted, they exert force on each other. Thus, the average interface pressure is the total force divided by the interface region. In contrast, pressure measurement is necessary to get a peak pressure when the interface pressure is not uniformly distributed. In this context, there are three technologies and methods to be considered to measure force or interface pressures, load cells, pressure indicating film, and tactile pressure sensor.

2.1. Load Cell. Load cell is a type of pressure sensor, which is commonly used in industrial weighing product to measure force such as goods and vehicles. The gripper of a robotic hand that picks up an object can be equipped with load cells in order to provide compression force feedback to the control system which prevents damage to the object or released too early. Also, load cells can be used to measure the compression forces during a robot walk to provide data for the equilibrium-controlling system. In industrial machinery, rods, beams, wheels, and bars are instrumented in order to control the forces exerted on them. Due to this variety of possible applications, load cells are very important [23]. There are many types of technologies which are used to measure loads such as strain gauges, piezoelectric elements, and variable capacitance.

Moreover, depending on the applied force and mechanics of application, multiple form factors of load cells are utilized. Typically, multiaxis MEMS force-torque sensors are used to measure the load. In the literature, a small number of multiaxis MEMS sensors have been reported. In [24],

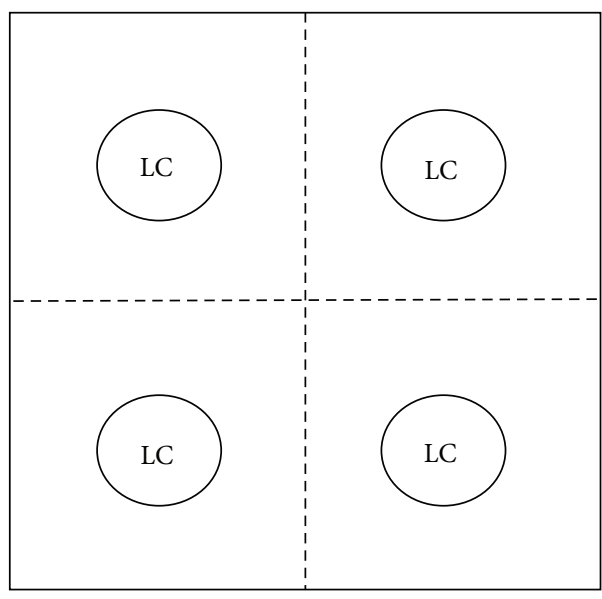

Figure 1: Pressure measurement using multiple load cells [30].

a capacitive MEMS force sensor is presented, which assess the force along two axes. In $[25,26]$, the design of a piezoresistive three-axis force sensor is described. A piezoresistive torque sensor has been presented in [27]. In addition, a three-axis capacitive MEMS force-torque sensor has been reported in [28] and this sensor is able to measure forces along two axes and a torque perpendicular to these forces. As we know, forces can be sensed in a plane, while a torque perpendicular to this plane can be measured. In certain conditions, researchers can use multiple load cells to measure forces over multiple regions of a contact interface [29]. In Figure 1, an interface or contact area is divided into four quadrants with an exclusive load cell measuring each area. This arrangement provides more details on the force distribution across the surface; the average pressure in each zone will occur. Still, the disadvantage of this technique is inconsistent because the load cell can show the total force but cannot identify localized spikes in pressure. There are many different types of load cells that operate in different ways, but currently the most commonly used load cell is the strain gage (or strain gauge) load cell.

2.1.1. Strain Gauge Load Cells. Strain gages are small patches of silicone or metal that measure mechanical strain and convert the load acting to electrical signals. This load cell is considered as an analog type tool and utilized to measure weight. When a load is applied to a stationary object, stress and strain are the result. Stress is defined as the object's internal resisting forces, and strain is the displacement and disfigurement that occurs [31], so the load causes deformations in the material or object that can be measured using strain gauges. Two capacitive pressure gauges which have been used extensively in the study of liquid and solid helium are described [32]. Figure 2 illustrates a structure of strain gauges. Here, more resistance in strain gauge will increase the stability as seen in Figure 2. In fact, it also offers a wide range of different patterns which means various applications will occur.

2.2. Pressure Indicating Film. Pressure indicating film is widely used to measure interface pressures between two 


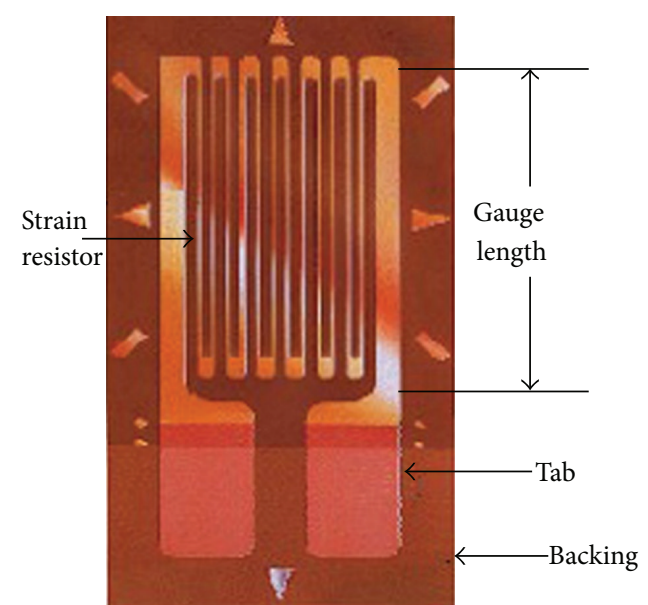

FIgURE 2: Structure of strain gauge [31].

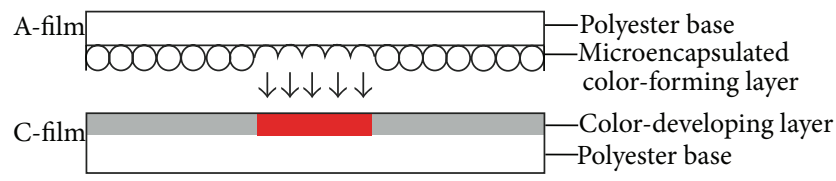

FIGURE 3: Components of pressure indicating film [30].

surfaces. Two sheets of polyester are designed to measure the force applied across the sensing area. Figure 3 shows a pressure indicating film. Here, a colour material, under a layer of polyester, is layered next to tiny microcapsules, which are utilized to break under different pressures. When pressure is applied to the film, the microcapsules are broken and distributed ink where pressure is deformed, and the colour intensity of the resulting image reveals the relative amount of applied pressure. As a result, the greater the pressure, the darker the colour and an image of the force applied will be composed across the sensing area. Various features cause the pressure indicating film to be used in a wide range of applications including flexibility, being easy to use, and thinness that plays a major role in capturing image of applied pressure. Furthermore, there are no attached electronics, that is, a good material or film to obtain the pressure distribution without concern of crushing wires or expensive electronics during the film feeding through rollers. Pressure indicating film is used in applications that requires static pressure measurements, visual pattern of peak pressure, and one-time use [30].

2.3. Tactile Pressure Sensor. Tactile pressure sensor measures various parameters of an object through physical touch between sensor and an object [16]. The measured parameters are, namely, pressure, temperature, normal and shear forces, vibrations, slip, and torques. In this context, pressure and torque are example of an important parameter, and it is typically measured through physical touch. Detection and measurement of a point contact force can also be considered as a part of touch sensor for pressure and torque, but then again, tactile sensing can also ease up the process

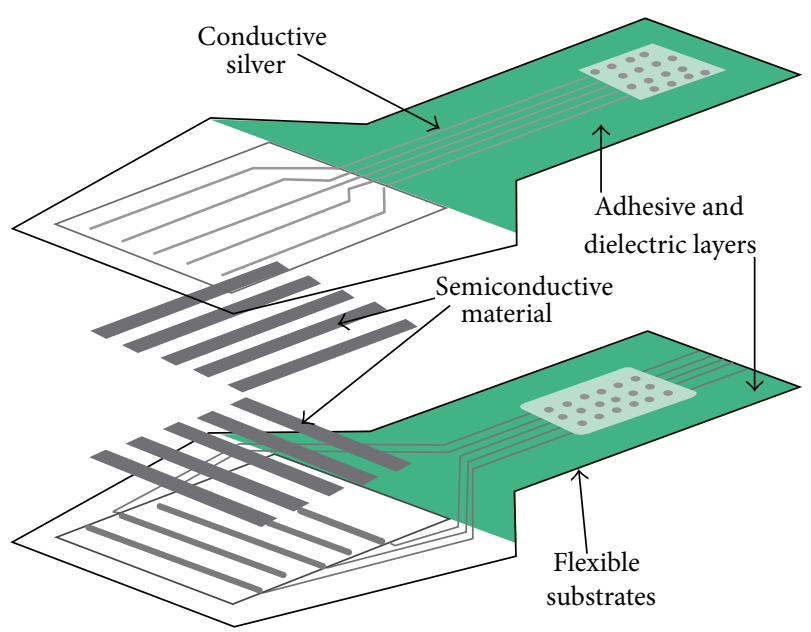

FIgURE 4: Construction of a tactile pressure sensor [30].

of interpretation of the corresponding information for the parameter.

By definition, tactile sensing means an array of a coordinated group of touch sensors [33]. A common type of tactile pressure sensor consists of an array sensor. For instance, Figure 4 shows a unique piezoresistive material sandwiched between two pieces of flexible polyester; each half of them has printed silver conductors. The result is a very thin $0.004^{\prime \prime}$ $(0.1 \mathrm{~mm})$ sensor, which can be used in various applications, especially for industrial and medical robot. A conductive track, which is composed of silver conductors, will scan the electronics and transmit a signal through the piezoresistive ink. The tactile array sensor signal can be processed to offer a great deal of parameters about contact kinematics and precise tactile information for robotics, haptic feedback, and other contact applications. Among the parameters that can be extracted are contact location, object shape, and effective width of the pressure distribution.

The pressure distribution can be achieved by identifying the position of all the applied forces. To do this, an array sensor which has vertical and horizontal of piezoresistive traces is needed. Here, each row and column intersecting in one point are called a sensel. More intersection implies more sensels which means the more spatial resolution is the sensor. Because of many human machine interfaces (e.g., wheelchair seating systems, driver's seats, bed mattresses, hospital beds) $[34,35]$ and because human joint is incongruent, the sensor should be in a wide range of resolutions. However, the tactile pressure sensor array has a good spatial resolution of the pressure distribution. Figure 5 illustrates the sensing system and an electrical schematic of electronics that scan each sensel. When force is applied to the sensel, the sensel which is represented by a variable resistor will be changed and the possible current will flow through the device, and then the electronics will collect the analog data, which can be compensated with proper calibration.

From the reviews that have been obtained, there are several factors to be considered, specifically on technology for interface force and pressure measurement between two 


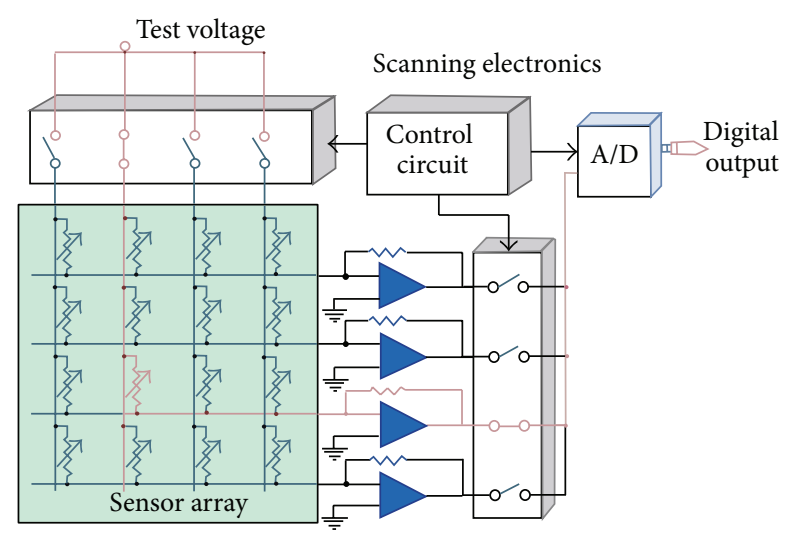

FIGURE 5: Electric schematic of sensor [30].

surfaces for robotic hand applications purposes. Comparisonwise, load cells provide the most reliable data pressure measurement, but the size and number of load cells limit the density of measurement points. The total load can be easily reported; however, the size of the load cell can be a limiting factor when it reaches fine granularity due to its pressure distribution.

Pressure indicating film can be used in variety of applications such as robotic hand, but the nature of the film will only provide the peak pressure between interfaces during a measurement. This has obvious limitations when trying to measure dynamic applications and also the resulting data pressure measurement has less accuracy, whereas tactile pressure sensor can provide detailed dynamic measurements of interface pressure with minimal impact on system dynamics. The sensing elements need to be properly calibrated to provide accurate data, but the resulting measurements will provide the most in-depth analysis of interface system dynamics. Depending on the information needed and the physical constraints of the system being measured, load cells, pressure indicating film, and tactile pressure sensors each have advantages and constraints for providing accurate and meaningful data pressure measurement. Understanding how these strengths and limitations influence an application is crucial.

\section{Pressure Sensor: Design and Technology}

A sensor is a device that measures a physical quantity and converts it into a signal which can be read by an observer or an instrument. There are various types of sensors: thermal sensor, electromagnetic sensor, mechanical sensor, pressure sensor, and others. Pressure is sensed by mechanical elements such as plates, shells, and tubes that are designed and constructed to deflect when pressure is applied. Deflection of the elements must be transduced to obtain an electrical or other output. Pressure sensor can differ in technology, design, performance, application suitability, and cost. It can be classified based on various transduction principles such as resistive/piezoresistive, tunnel effect, capacitive, optical, ultrasonic, magnetic, and piezoelectric. The relative merits

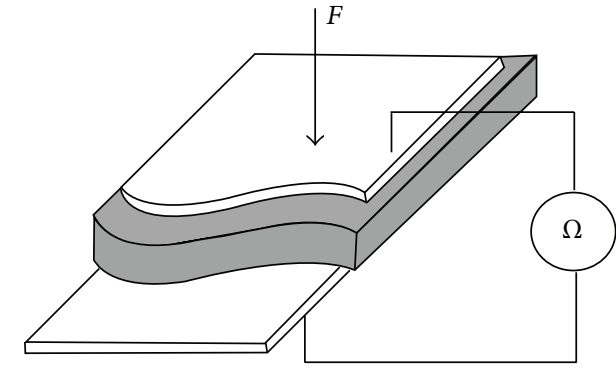

FIGURE 6: Piezoresistive tactile pressure sensor [38].

and demerits of different transduction methods are given in Table 1. Worldwide, there are hundreds of different technologies used in pressure sensor designs such as sensing element method, material, MEMS, nanotechnology, and others. Furthermore, there are significant differences in the types of pressure sensor results from different material used as well as their functional properties.

Recently, pressure sensor using MEMS technology has received enormous attention due to various advantages over traditional electromechanical sensing technology. MEMS offers small size, low weight, low cost, high performance, large scale integration, low power consumption, wider operating temperature, and higher output signal [36]. This section discusses in detail the three main types of pressure sensor methods for robotic hand application: piezoresistive, piezoelectric, and capacitive pressure sensor. Also, various designs and technologies of the pressure sensor are explained in this section.

3.1. Piezoresistive FlexiForce Sensor. As sensor technology grows these days, there are many types of sensors that have been used for pick and place application, especially resistive method due to its stability and high sensitivity. Piezoresistive sensors use the change of the electrical resistance in a material when it has been mechanically deformed. The resistance of a piezoresistor is given as follows:

$$
R=\frac{\rho \times l}{t \times \omega},
$$

where $\rho, l, t$, and $\omega$ denote the resistivity, length, thickness of the piezoresistor, and the width of the contact, respectively. Figure 6 shows an example of the piezoresistive tactile pressure sensor. Due to the various features of piezoresistive including low cost, good sensitivity, relatively simple construction, long-term stability with low noise, accuracy, and reliability, it shows the maturity of the technology. In addition, the sensor is considered easy to fabricate and integrate with electronic circuit according to the characteristic of the piezoresistive material. However, it can measure only one contact location and it will still need external power. Though this limitation has been improved by [37], that allows measuring many contact points by using parallel analog resistive sensing strips.

The force sensing resistor (FSR) is based on piezoresistive sensing technology. It can be made in a variety of shapes and 
TABLE 1: Relative merits and demerits of various tactile sensor types [17].

\begin{tabular}{|c|c|c|}
\hline Type & Merits & Demerits \\
\hline Resistive & $\begin{array}{l}\text { (i) Sensitive } \\
\text { (ii) Low cost }\end{array}$ & $\begin{array}{l}\text { (i) High power consumption } \\
\text { (ii) Generally detect single contact point } \\
\text { (iii) Lack of contact force measurement }\end{array}$ \\
\hline Piezoresistive & $\begin{array}{l}\text { (i) Low cost } \\
\text { (ii) Good sensitivity } \\
\text { (iii) Low noise } \\
\text { (iv) Simple electronics }\end{array}$ & $\begin{array}{l}\text { (i) Stiff and frail } \\
\text { (ii) Nonlinear response } \\
\text { (iii) Hysteresis } \\
\text { (iv) Temperature sensitive }\end{array}$ \\
\hline Tunnel effect & $\begin{array}{l}\text { (i) Sensitive } \\
\text { (ii) Physically flexible }\end{array}$ & (i) Nonlinear response \\
\hline Capacitive & $\begin{array}{l}\text { (i) Sensitive } \\
\text { (ii) Low cost } \\
\text { (iii) Availability of commercial A/D chips }\end{array}$ & $\begin{array}{l}\text { (i) Hysteresis } \\
\text { (ii) Complex electronics }\end{array}$ \\
\hline Optical & $\begin{array}{l}\text { (i) Physically flexible } \\
\text { (ii) Sensitive } \\
\text { (iii) Fast } \\
\text { (iv) No interconnections }\end{array}$ & $\begin{array}{l}\text { (i) Loss of light by micro bending chirping } \\
\text { (ii) Power consumption } \\
\text { (iii) Complex computations }\end{array}$ \\
\hline Ultrasonic & $\begin{array}{l}\text { (i) Fast dynamic response } \\
\text { (ii) Good force resolution }\end{array}$ & $\begin{array}{l}\text { (i) Limited utility at low frequency } \\
\text { (ii) Complex electronics } \\
\text { (iii) Temperature sensitive }\end{array}$ \\
\hline Magnetic & $\begin{array}{l}\text { (i) High sensitivity } \\
\text { (ii) Good dynamic range } \\
\text { (iii) No mechanical hysteresis } \\
\text { (iv) Physical robustness }\end{array}$ & $\begin{array}{l}\text { (i) Suffer from magnetic interference } \\
\text { (ii) Complex computations } \\
\text { (iii) Somewhat bulky } \\
\text { (iv) Power consumption }\end{array}$ \\
\hline Piezoelectric & $\begin{array}{l}\text { (i) Dynamic response } \\
\text { (ii) High bandwidth }\end{array}$ & $\begin{array}{l}\text { (i) Temperature sensitive } \\
\text { (ii) Not so robust electrical connection }\end{array}$ \\
\hline Conductive rubber & (i) Physically flexible & $\begin{array}{l}\text { (i) Mechanical hysteresis } \\
\text { (ii) Nonlinear response }\end{array}$ \\
\hline
\end{tabular}

sizes and can be utilized in many applications in order to measure a proportional change in force and rate of change and also detects contact or touch between objects. FlexiForce manufactured Tekscan is one of the most piezoresistive sensors widely used in robotic hand. Figure 7 shows a tactile force sensor or FlexiForce sensors. This sensor is considered one of the best ideal force sensors for designers, researchers, or anyone who needs to measure forces. With its thin construction, flexibility, and force measurement ability, the FlexiForce sensor can measure the force between any two surfaces and is resilient to most environments. FlexiForce has better force sensing properties, linearity, low hysteresis, drift, and temperature sensitivity than any other thin film force sensors according to the good substrate material which is a polymer. This material has been considered suitable enough to use in robotic hand for grasping objects effectively.

The structure of the force sensor is a substitute of a matrix of sensing traces; the ink uniformly covers an area to measure the total force applied to that space. The sensor consists of two layers of substrate as shown in Figure 7. This substrate is formed of polyester film and a conductive material, silver, which applies to each layer. Layer of pressure sensitive ink is then used, followed by adhesive to combine the two layers of substrate together to compose the sensor. Additionally, the FlexiForce sensor decreases the resistance of the sensing element when the force applied increases. In this context, various applications using FlexiForce sensor are implemented by many researchers [39]. As an example, measurement of interface pressure or force between two soft objects is presented in [40]. Teleoperated robotic systems using tactile force sensor for the design and development of a low cost control rig to intuitively manipulate an anthropomorphic robotic arm with gripping force sensing are reported in [41]. The measurement of low interface pressure between the skin support surfaces and pressure garments is also discussed in [42]. Thereupon, one good example of using FlexiForce sensors is pick and place application which offers to achieve high sensitivity and minimize slip movement and weight measurement with a secure grasp.

3.2. Piezoelectric Pressure Sensor. Piezoelectric sensors convert an applied stress or force into an electric voltage [43]. Piezoelectric material is considered a smart material due to its property which can be used as a sensor and actuators. Furthermore, the piezoelectric materials also have high sensitivity with high voltage output when force is applied. The sensitivity of piezoelectric force sensors is measured in terms of $\mathrm{C} / \mathrm{N}$, with sensitivity reported up to $130 \mathrm{pC} / \mathrm{N}$ [39]. Piezoelectric is considered as a passive sensor which offers a high reliability that is useful to be applied in various applications. Yet, it is only suitable for detection of dynamic forces because of the voltage output decreases over time [44], because it is not able to measure a static force due to their large internal resistance [45]. Piezoelectric materials like 


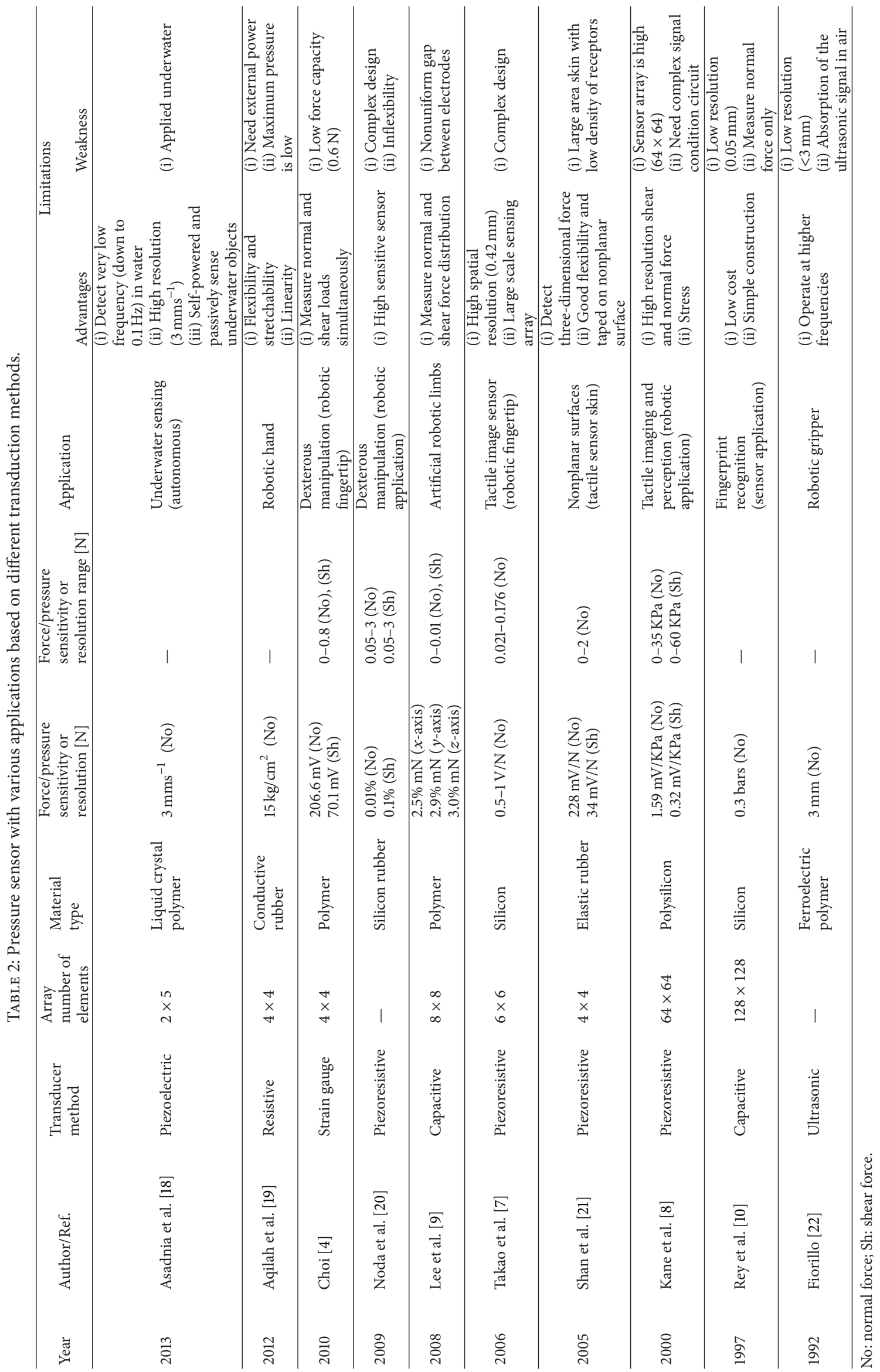




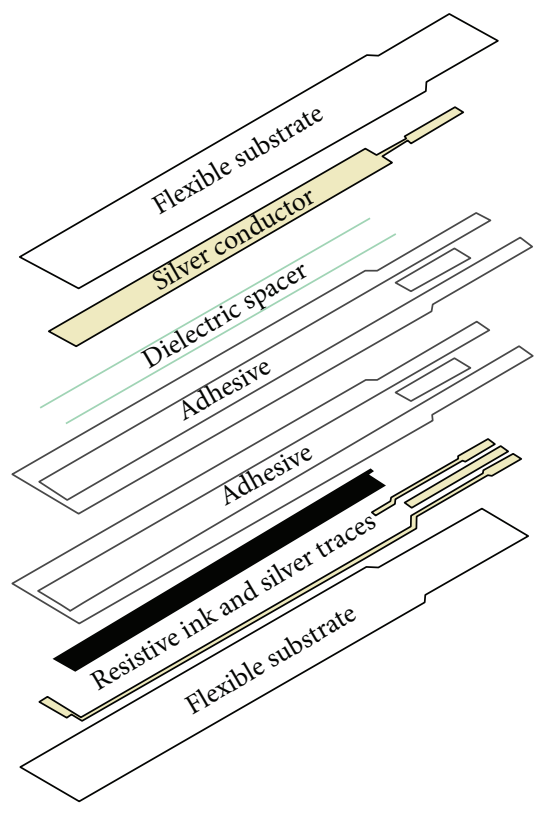

Figure 7: Components of FlexiForce sensor [30].

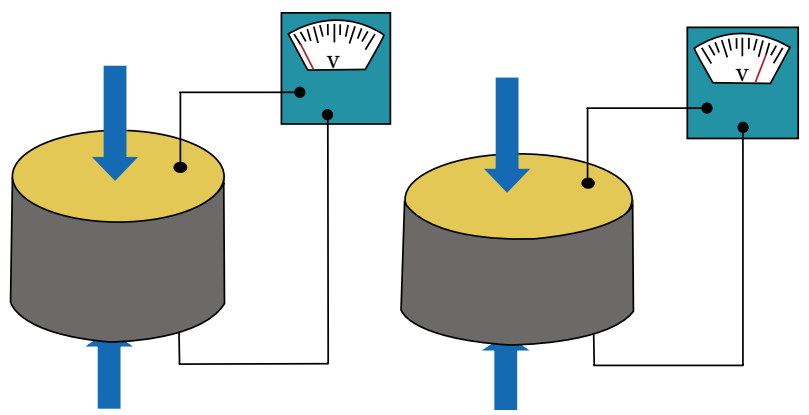

Figure 8: The behaviour of piezoelectric disk based on pressure forces [30].

ceramic lead zirconate titanate (PZT), polymer polyvinylidene fluoride (PVDF), and so forth are suitable for dynamic tactile sensing. Although quartz and ceramic PZT have better piezoelectric properties, the polymer PVDF is more preferred in touch sensors because of their excellent features including mechanical flexibility, high piezoelectric coefficients, dimensional stability, low weight, workability, chemical stability, and chemical inertness [46-48]. The first time PVDF was implemented for tactile sensing technology was reported in [49] and, recently, it was used for environment perception as discussed in [50]. Henceforth, to design the circuit of piezoelectric sensor, an ultrathin input impedance is needed as a considerable effect on response to the device. Figure 8 illustrates that a piezoelectric disk generates a voltage when deformed.

3.3. Capacitive Pressure Sensor. Capacitive sensors consist of a plate capacitor, in which the distance between the plates or electrode area is changed when compressed, and it has a suspended structure that can measure the change in

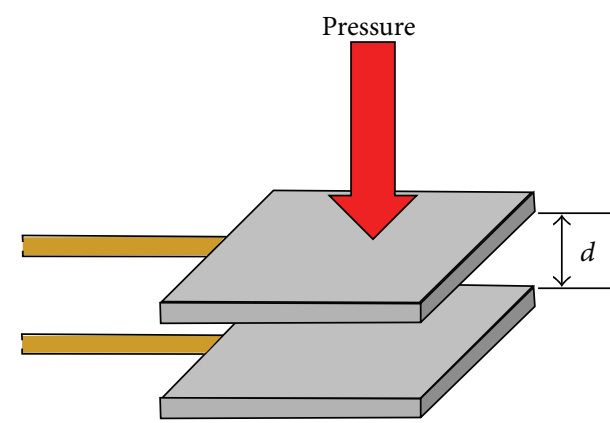

FIGURE 9: Capacitive sensor with two parallel plates [30].

the capacitance between these two electrodes. For parallel plate capacitors, capacitance can be expressed as follows:

$$
C=\frac{\varepsilon o K A}{d},
$$

where $C$ is the capacitance, $\varepsilon o$ is the relative permittivity of free space constant, $K$ is the dielectric constant of the material in the gap, $A$ is the area of the plates, and $d$ is the distance between the plates. Capacitive tactile pressure sensing is considered as one of the most sensitive techniques for detecting small deflections of structures [51]. It has been developed by researchers for many years due to its features having high spatial resolution, good frequency response, low power consumption, and a large dynamic range [45]. A capacitor sensor array is introduced in [52] and fabricated directly on flexible thin films of polyamide with thickness $25 \mu \mathrm{m}$. The sensors show a linear response to applied pressure. Also, few instances of capacitive touch sensors are presented in [53]. Subsequently, an $8 \times 8$ capacitive tactile sensing array with spatial resolution at least 10 times better than the human limit of $1 \mathrm{~mm}$ is reported in [54]. Two electrodes with air gap, $d$, are shown in Figure 9.

As summarised, there is no ideal pressure sensor technology that can be used in all applications, since each has specific advantages and constraints. As a matter of fact, the pressure sensor design is primarily determined by the application requirements. It is not just the pressure sensor technology that is vital, but also the practicalities of its implementation of the pressure sensor design must be considered. Furthermore, a wide variety of materials and technologies has been used for the pressure sensor, resulting in performance versus cost tradeoffs. The electrical output signal also provides a variety of choices for various applications.

\section{Robotic Hand: Applications}

As we know, the human hand is one of the most important parts in a body as it can arrive at narrow places and can execute complex operations. Hence, it is essential for us to have a robotic hand which can accomplish the same procedure as a human hand does in real time. Back in the days, the abilities of humanoid robots focused on walking. Only just, some robotic hands have been developed and there has been a mounting interest in supplying them with 


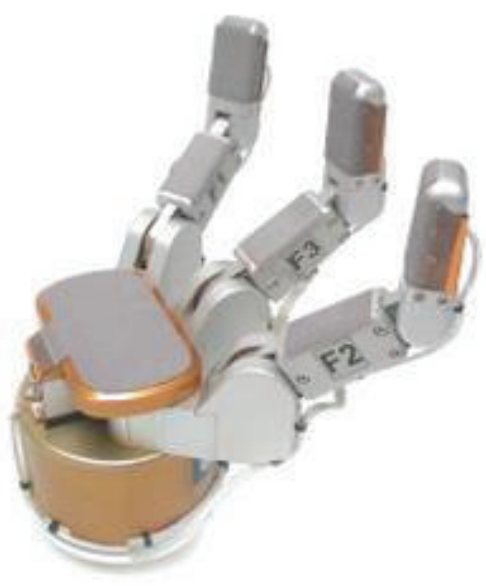

FIGURE 10: Robotic hand with tactile sensor [33].

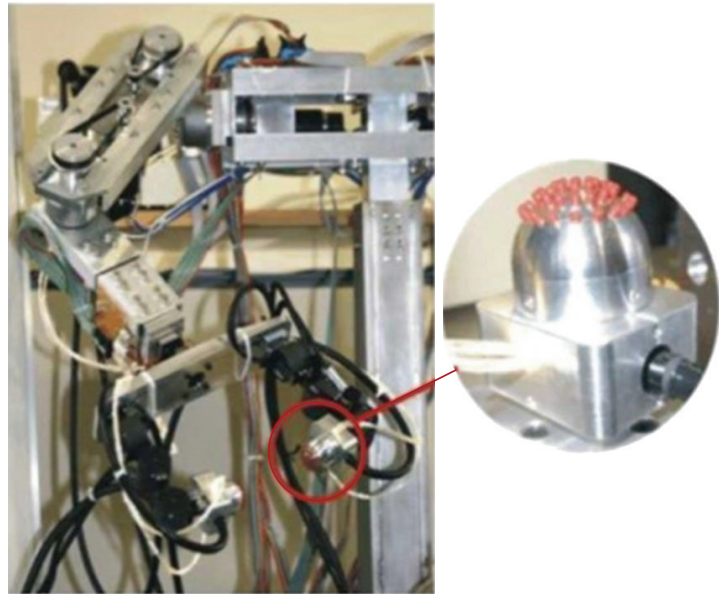

FIgURE 11: Optical three-axis tactile sensor [64]. proceeding manipulation capabilities [55-58]. Robotic hands have a lot of technologies to execute depending on the required applications, in some instances, picking and sorting cookies [59], military robots [60], welding robots [61], and also nuclear industry robots [62]. Likewise, robotic hands can also be found in various fields such as manufacturing industry, military, space exploration, domestic, transportation, and medical applications.

In this context, manipulation capabilities are one of the robotic hand applications that are central to a robot system. Figure 10 shows an example using commercial products, a robotic hand with tactile sensors from Pressure Profile Systems, Inc. (PPS). This tactile sensing technology gives the robotic hand the ability to manipulate delicate objects without breaking them. Moreover, they will also be able to operate at optimized low powers for energy efficiency by using minimized grasp force. Robotic platform using capacitive sensors is also produced by Pressure Profile Systems, Inc., and it is described in [63].

Next, optical three-axis tactile sensors are shown in Figure 11. It is used to improve sensitization quality in robotic hand system [64]. The arm actuators use the tactile information as feedback to execute and request the orders as needed.

4.1. Tactile Transduction Techniques and Applications. Tactile pressure sensor has been mulled over to be one of the suitable pressure sensors that allow humans to execute dexterous manipulation and offer robot manipulators (hands/fingers) with accurate information on the objects to grab, hold, and handle. Dexterous robotic hands have been developed for the purpose of grasping different objects and it is very challenging for many researchers $[65,66]$. Over the years, the change from structured to unstructured environments has made the development of different sensors a priority to enable robots to cope with considerable uncertainties. Because of that, sensors that can get back tactile information have been developed in order to prepare robot hands with such a sense [67].
There are many tactile pressure sensors that are based on a variety of principles such as resistive, capacitive, optical, ultrasonic, magnetic, piezoresistive, or piezoelectric sensors. Up to date, a lot of tactile pressure sensors have been developed. Some of these works, classified on the basis of sensitivity, range of pressure, type of force, and resolution, are given in Table 2. From 1992 to 2013 as viewed in Table 2, methods miscellaneous technologies with various types of materials have been used to sense the pressure in a wide range of applications, particularly in robotic hand. During the time, the size of the sensor design becomes small and delicate. In comparison, an array of $64 \times 64$ elements was used in tactile imaging and perception with piezoresistive transducer in 2000 as shown in Table 2. On the other hand, after decade of time, technology would have the potential to support the development of more intelligent products in order to improve the quality of human life as in 2012. In this year, many researchers involve their products to achieve what human life needed; a good example is a $4 \times 4$ array small in size and conformable using the same transducer method comparing with previous example but in a different type of material which is conductive rubber. Additionally, tactile pressure sensor can measure both normal and shear forces produced through dexterous manipulation $[68,69]$. However, most of existing tactile sensors only detect the normal contact force during handling objects in a robotic hand manipulation. Hence, the measure of shear force is as important as a normal force to emulate the human hand, which can simultaneously sense the direction and the strength of the applied force within sophisticated manipulation. Likewise, measure of the mechanical contact forces allows controlling the grasp force, which is essential for manipulator displacement and slip movements. This slip detection between fingertip and objects is still one of the main issues that researchers dedicated their time to put an optimal solution to. In fact, this matter required analysing and measuring both shear and normal forces. For this reason, shear information is considered of great importance to full grasp force and torque determination, especially when the pressure does not exceed more than $0.1 \mathrm{~N}$ [4]. 
These parameters will be used to predict and determine slipping of the object.

4.2. Pick and Place Applications. Robotic hands are widely used in manufacturing industry. Typically, it is used for pick and place robot (such as packaging and palletizing). Pick and place process requires the use of robotic hands in order to manipulate objects. In this field, robotic hands have to be programmed in a familiar environment to avoid probable conflict between tools and objects. The agricultural robotic hand is also considered to be a good example to ease up a farmer's job in cutting grass [70]. This robot, which contains a manipulator, a visual sensor, a travelling device, and end-effectors, is able to do a number of tasks by changing the end-effectors. Painting robotic is provided with a door handler of the arm for opening and closing a vehicle door before and after painting it [71]. Furthermore, this system provides a robot for automatic painting vehicle bodies and reduces the equipment's cost by performing the operations of painting vehicle bodies by itself. Similarly, a robot laser welding system is a robot system that consists of a servocontrolled, multiaxis mechanical hand, with a laser cutting head mounted to the faceplate of the robotic hand. The cutting head has focused optics for the laser light and an integral height control mechanism. Most systems use a laser generator that conveys the laser light to the robot cutting head through an optical fiber cable. The benefits of laser welding are that it will produce higher productivity, improved flexibility, and quality welds $[72,73]$. All of existing applications used a robotic hand, which are equipped by diverse kinds of sensors to carry through the tasks as necessitated. As a consequence, these missions cannot be accomplished as required without algorithm, since it will be as a framework for the mechanism of robotic hand. Likewise, high performance and safe operating will occur within this algorithm.

4.3. Control Algorithm. From recent development, it is traced that manipulation control is important for a robot. Manipulation control requires some kind of feedback which could provide information about the interaction between the gripper and the grasp objects. This feedback information can be used to implement an algorithm control to achieve the function operator of any robotic hand application as required. It has also been reported that multifingered robotic hand executes particular tasks of grasping an object, which needs to control the measuring required forces for successful operation and dexterous gripper. In addition, it can grasp various objects by changing its shape. Nonetheless, in many cases they lack linearity or sensitivity, especially, in terms of masterful gripper [74]. The robotic hand gripper can increase the sensitivity as well as linearity by using an intelligent feedback control which will be doing the mechanism of gripper object effectively. To wrap up everything, various robotic hand applications using the different algorithm control had been discussed based on tactile sensing capabilities to increase accuracy, flexibility, and receptivity. Moreover, in future robotic hand applications, the manipulators will have to be made lighter and move faster with higher accuracy and work

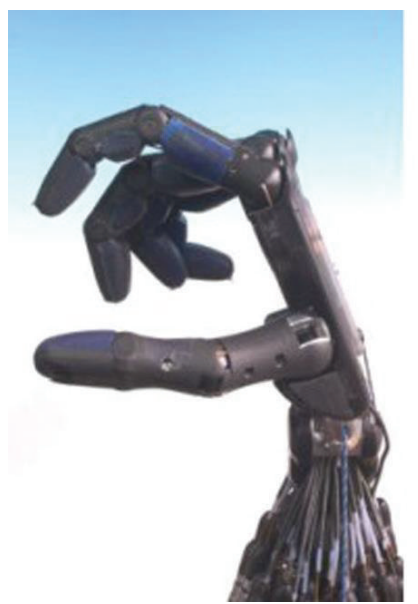

Figure 12: The Shadow Dextrous Robot Hand [77].

independently. For instance, the automation of complex tasks in industrial applications would be highly enhanced if robots could operate at high speed with high accuracy. Nevertheless, the current robot designs are made massive in size in order to increase rigidity; thereupon, these aims cannot be executed. To achieve high speed operation and faster response for robotic hands manipulations, we should reduce the driving torque requirement. For this purpose, many one-arm flexible robot arms have been built in laboratories $[75,76]$. The Shadow Dextrous Robot Hand, in Figure 12, is an advanced humanoid robot hand system available for purchase and is regarded as one of the most advanced robot hands in the current world.

In a nutshell, various factors play an important role in achieving the appropriate application as necessitated including material type, transduction method, and conditioning circuit. These factors set the limitation of every application as viewed in Table 2. For instance, the polymer (polyimide) utilized in the tactile pressure sensor fabrication is used for dexterous robotic manipulation applications such as grasping objects, due to it is advantages which are flexible as well as robust enough to withstand forces during grasping. Differently, the silicon MEMS technology used in a tactile image and recognition in robotic applications requires less flexibility but needs high spatial resolution and sensitivity. Hence, the major application of polymer can be applied in wide area tactile sensor like artificial skin and nonplanar surface considered to be having lower fabrication cost than silicon. Moreover, silicon MEMS also reduces the number of electronic signal wires which make it suitable for fingertip and image recognition applications that need high resolution and also suitable for flat surface.

Beside this, the best choice of transduction method and conditioning circuit is very important as they set the limit of power consumption, time response, and number of sensors, allowed to be used in an array. Yet, although piezoresistive sensors are commonly sensitive and economic, they still consume a lot of power rather than others. In addition, they are suitable for detecting dynamic force but have a limitation in the robotic hand application due to voltage 
output decreases over time and large internal resistance which make them not able to measure a static force.

\section{Conclusion}

In this paper, different techniques of pressure sensor types have been reviewed including load cell, pressure indicating film, and tactile pressure mapping system. Similarly, various transaction methods, including piezoresistive, capacitive, and piezoelectric are discussed. Tactile pressure sensor based on piezoresistive material for robotic hand application is also presented. Different materials are used to sense the pressure in many applications including conductive rubber, elastic cantilevers, swollen silicon, elastic rubber, polysilicon, ferroelectric, and polymer. Recently, piezoresistive methods were used for robotic designed especially on grasping objects. It was defined that the advantages were due to making use of polymers which are more flexible, linear, and stretchable. Therefore, it was found that the risk process to workers was reduced and increased safety between the robotic hand and the object during the interaction process.

\section{Conflict of Interests}

The authors declare that there is no conflict of interests regarding the publication of this paper.

\section{Acknowledgments}

This research was supported by a Science Fund grant from the Malaysian Ministry of Science, Technology and Innovation and Grant Putra from the Universiti Putra Malaysia.

\section{References}

[1] G. Song, H. Wang, J. Zhang, and T. Meng, "Automatic docking system for recharging home surveillance robots," IEEE Transactions on Consumer Electronics, vol. 57, no. 2, pp. 428-435, 2011.

[2] L. Zollo, S. Roccella, E. Guglielmelli, M. C. Carrozza, and P. Dario, "Biomechatronic design and control of an anthropomorphic artificial hand for prosthetic and robotic applications," IEEE/ASME Transactions on Mechatronics, vol. 12, no. 4, pp. 418-429, 2007.

[3] A. Rodriguez, M. T. Mason, and S. S. Srinivasa, "Manipulation capabilities with simple hands," in Experimental Robotics, vol. 79 of Springer Tracts in Advanced Robotics, pp. 285-299, Springer, Berlin, Germany, 2014.

[4] W.-C. Choi, "Polymer micromachined flexible tactile sensor for three-axial loads detection," Transactions on Electrical and Electronic Materials, vol. 11, no. 3, pp. 130-133, 2010.

[5] E. L. Lawrence, I. Fassola, S. Dayanidhi, C. Leclercq, and F. J. Valero-Cuevas, "An evaluation of clustering techniques to classify dexterous manipulation of individuals with and without dysfunction," in Proceedings of the 6th International IEEE/EMBS Conference on Neural Engineering (NER '13), pp. 1254-1257, November 2013.

[6] M. Florek-Jasinska, T. Wimbock, and C. Ott, "Humanoid compliant whole arm dexterous manipulation: control design and experiments," in Proceedings of the IEEE/RSJ International
Conference on Intelligent Robots and Systems (IROS '14), pp. 1616-1621, Chicago, Ill, USA, September 2014.

[7] H. Takao, K. Sawada, and M. Ishida, "Monolithic silicon smart tactile image sensor with integrated strain sensor array on pneumatically swollen single-diaphragm structure," IEEE Transactions on Electron Devices, vol. 53, no. 5, pp. 1250-1259, 2006.

[8] B. J. Kane, M. R. Cutkosky, and G. T. A. Kovacs, "A traction stress sensor array for use in high-resolution robotic tactile imaging," Journal of Microelectromechanical Systems, vol. 9, no. 4, pp. 425434, 2000.

[9] H.-K. Lee, J. Chung, S.-I. Chang, and E. Yoon, "Normal and shear force measurement using a flexible polymer tactile sensor with embedded multiple capacitors," Journal of Microelectromechanical Systems, vol. 17, no. 4, pp. 934-942, 2008.

[10] P. Rey, P. Charvet, M. T. Delaye, and S. A. Hassan, "High density capacitive pressure sensor array for fingerprint sensor application," in Proceedings of the International Conference on Solid-State Sensors and Actuators, pp. 1453-1456, Chicago, Ill, USA, June 1997.

[11] S. Hirose and Y. Umetani, "The development of soft gripper for the versatile robot hand," Mechanism and Machine Theory, vol. 13, no. 3, pp. 351-359, 1978.

[12] V. Lippiello, F. Ruggiero, B. Siciliano, and L. Villani, "Visual grasp planning for unknown objects using a multifingered robotic hand," IEEE/ASME Transactions on Mechatronics, vol. 18, no. 3, pp. 1050-1059, 2013.

[13] F. Suárez-Ruiz, I. Galiana, Y. Tenzer, L. P. Jentoft, R. D. Howe, and M. Ferre, "Grasp mapping between a 3-finger haptic device and a robotic hand," in Haptics: Neuroscience, Devices, Modeling, and Applications, Lecture Notes in Computer Science, pp. 275283, Springer, Berlin, Germany, 2014.

[14] R. H. Cushman and C. L. Hoegermeyer, "Vacuum pick and place robotic hand," Google Patents, 1985.

[15] Y. Zhang, B. K. Chen, X. Liu, and Y. Sun, "Autonomous robotic pick-and-place of microobjects," IEEE Transactions on Robotics, vol. 26, no. 1, pp. 200-207, 2010.

[16] M. H. Lee and H. R. Nicholls, "Review Article Tactile sensing for mechatronics-a state of the art survey," Mechatronics, vol. 9, no. 1, pp. 1-31, 1999.

[17] R. S. Dahiya and M. Valle, "Tactile sensing for robotic applications," in Sensors: Focus on Tactile, Force and Stress Sensors, pp. 289-304, InTech, 2008.

[18] M. Asadnia, A. G. P. Kottapalli, Z. Shen, J. Miao, and M. Triantafyllou, "Flexible and surface-mountable piezoelectric sensor arrays for underwater sensing in marine vehicles," IEEE Sensors Journal, vol. 13, no. 10, pp. 3918-3925, 2013.

[19] A. Aqilah, A. Jaffar, S. Bahari, C. Y. Low, and T. Koch, "Resistivity characteristics of single miniature tactile sensing element based on pressure sensitive conductive rubber sheet," in Proceedings of the IEEE 8th International Colloquium on Signal Processing and Its Applications (CSPA '12), pp. 223-227, March 2012.

[20] K. Noda, Y. Hashimoto, Y. Tanaka, and I. Shimoyama, "MEMS on robot applications," in Proceedings of the International SolidState Sensors, Actuators and Microsystems Conference (TRANSDUCERS '09), pp. 2176-2181, Denver, Colo, USA, June 2009.

[21] J. H. Shan, T. Mei, L. Sun, L. Ni, M. Meng, and J. R. Chu, "The design and fabrication of a flexible three-dimensional force sensor skin," in Proceedings of the IEEE/RSJ International Conference on Intelligent Robots and Systems (IROS '05), pp. 1818-1823, August 2005. 
[22] A. S. Fiorillo, "Design and characterization of a PVDF ultrasonic range sensor," IEEE Transactions on Ultrasonics, Ferroelectrics, and Frequency Control, vol. 39, no. 6, pp. 688-692, 1992.

[23] I. Muller, R. de Brito, C. E. Pereira, and V. Brusamarello, "Load cells in force sensing analysis-theory and a novel application," IEEE Instrumentation and Measurement Magazine, vol. 13, no. 1, pp. 15-19, 2010.

[24] Y. Sun, B. J. Nelson, D. P. Potasek, and E. Enikov, "A bulk microfabricated multi-axis capacitive cellular force sensor using transverse comb drives," Journal of Micromechanics and Microengineering, vol. 12, no. 6, pp. 832-840, 2002.

[25] J. Brugger, M. Despont, C. Rossel, H. Rothuizen, P. Vettiger, and M. Willemin, "Microfabricated ultrasensitive piezoresistive cantilevers for torque magnetometry," Sensors and Actuators A: Physical, vol. 73, no. 3, pp. 235-242, 1999.

[26] W. L. Tin and C. D. Mote Jr., "Development and calibration of a sub-millimeter three-component force sensor," Sensors and Actuators A: Physical, vol. 65, no. 1, pp. 89-94, 1998.

[27] S. Bütefisch, S. Büttgenbach, T. Kleine-Besten, and U. Brand, "Micromechanical three-axial tactile force sensor for micromaterial characterisation," Microsystem Technologies, vol. 7, no. 4, pp. 171-174, 2001.

[28] F. Beyeler, S. Muntwyler, Z. Nagy, M. Moser, and B. J. Nelson, "A multi-axis MEMS force-torque sensor for measuring the load on a microrobot actuated by magnetic fields," in Proceedings of the IEEE/RSJ International Conference on Intelligent Robots and Systems (IROS '07), pp. 3803-3808, San Diego, Calif, USA, November 2007.

[29] J. L. Tan, J. Tien, D. M. Pirone, D. S. Gray, K. Bhadriraju, and C. S. Chen, "Cells lying on a bed of microneedles: an approach to isolate mechanical force," Proceedings of the National Academy of Sciences of the United States of America, vol. 100, no. 4, pp. 1484-1489, 2003.

[30] Tekscan, "FlexiForce Force Sensors," September 2014, http://www.tekscan.com/interface-pressure-measurementoptions.

[31] J. I. Bae, T. H. An, Y. S. Kim, and C. K. Ryu, "Analysis of digital load cell using $2.4 \mathrm{GHz}$ band's Zig-Bee," in Proceedings of the 3rd IEEE Conference on Industrial Electronics and Applications (ICIEA '08), pp. 1358-1361, June 2008.

[32] E. D. Orth, "Semiconductor strain gage pressure transducer," Google Patents, 1972.

[33] P. S. Girão, P. M. P. Ramos, O. Postolache, and J. M. D. Pereira, "Tactile sensors for robotic applications," Measurement, vol. 46, no. 3, pp. 1257-1271, 2013.

[34] D. E. Gyi, J. M. Porter, and N. K. B. Robertson, "Seat pressure measurement technologies: considerations for their evaluation," Applied Ergonomics, vol. 29, no. 2, pp. 85-91, 1998.

[35] X. Wu, S. Rakheja, and P.-É. Boileau, "Distribution of humanseat interface pressure on a soft automotive seat under vertical vibration," International Journal of Industrial Ergonomics, vol. 24, no. 5, pp. 545-557, 1999.

[36] F. Khoshnoud and C. W. de Silva, "Recent advances in MEMS sensor technology-biomedical applications," IEEE Instrumentation and Measurement Magazine, vol. 15, no. 1, pp. 8-14, 2012.

[37] H. Zhang and E. So, "Hybrid resistive tactile sensing," IEEE Transactions on Systems, Man, and Cybernetics Part B: Cybernetics, vol. 32, no. 1, pp. 57-65, 2002.

[38] K. Weiß and H. Worn, "The working principle of resistive tactile sensor cells," in Proceedings of the IEEE International Conference on Mechatronics and Automation, pp. 471-476, 2005.
[39] A. M. M. Almassri, W. Z. W. Hasan, S. A. Ahmad, and A. J. Ishak, "A sensitivity study of piezoresistive pressure sensor for robotic hand," in Proceedings of the IEEE Regional Symposium on Micro and Nano Electronics (RSM '13), pp. 394-397, Langkawi, Malaysia, September 2013.

[40] C. M. A. Ashruf, “Thin flexible pressure sensors," Sensor Review, vol. 22, no. 4, pp. 322-327, 2002.

[41] G. S. Gupta, S. C. Mukhopadhyay, C. H. Messom, and S. N. Demidenko, "Master-slave control of a teleoperated anthropomorphic robotic arm with gripping force sensing," IEEE Transactions on Instrumentation and Measurement, vol. 55, no. 6, pp. 2136-2145, 2006.

[42] M. Ferguson-Pell, S. Hagisawa, and D. Bain, "Evaluation of a sensor for low interface pressure applications," Medical Engineering and Physics, vol. 22, no. 9, pp. 657-663, 2000.

[43] C. Steinem, A. Janshoff, and M. A. Cooper, Piezoelectric Sensors, Springer, New York, NY, USA, 2007.

[44] P. Puangmali, K. Althoefer, L. D. Seneviratne, D. Murphy, and P. Dasgupta, "State-of-the-art in force and tactile sensing for minimally invasive surgery," IEEE Sensors Journal, vol. 8, no. 4, pp. 371-380, 2008.

[45] M. I. Tiwana, S. J. Redmond, and N. H. Lovell, "A review of tactile sensing technologies with applications in biomedical engineering," Sensors and Actuators A: Physical, vol. 179, pp. 1731, 2012.

[46] J. R. Flanagan and A. M. Wing, "Modulation of grip force with load force during point-to-point arm movements," Experimental Brain Research, vol. 95, no. 1, pp. 131-143, 1993.

[47] S. B. Lang and S. Muensit, "Review of some lesser-known applications of piezoelectric and pyroelectric polymers," Applied Physics A, vol. 85, no. 2, pp. 125-134, 2006.

[48] A. Song, Y. Han, H. Hu, and J. Li, "A novel texture sensor for fabric texture measurement and classification," IEEE Transactions on Instrumentation and Measurement, vol. 63, no. 7, pp. 17391747, 2013.

[49] P. Dario and D. De Rossi, "Tactile sensors and the gripping challenge: increasing the performance of sensors over a wide range of force is a first step toward robotry that can hold and manipulate objects as humans do," IEEE Spectrum, vol. 22, no. 8, pp. 46-53, 1985.

[50] A. Song, Y. Han, H. Hu, L. Tian, and J. Wu, "Active perceptionbased haptic texture sensor," Sensors and Materials, vol. 25, no. 1, pp. 1-15, 2013.

[51] V. Maheshwari and R. Saraf, "Tactile devices to sense touch on a par with a human finger," Angewandte Chemie International Edition, vol. 47, no. 41, pp. 7808-7826, 2008.

[52] E. Pritchard, M. Mahfouz, B. Evans, S. Eliza, and M. Haider, "Flexible capacitive sensors for high resolution pressure measurement," in Proceedings of the Sensors Conference, pp. 14841487, Lecce, Italy, 2008.

[53] P. A. Schmidt, E. Maël, and R. P. Würtz, "A sensor for dynamic tactile information with applications in human-robot interaction and object exploration," Robotics and Autonomous Systems, vol. 54, no. 12, pp. 1005-1014, 2006.

[54] B. L. Gray and R. S. Fearing, "A surface micromachined microtactile sensor array," in Proceedings of the IEEE International Conference on Robotics and Automation, vol. 1, pp. 1-6, Minneapolis, Minn, USA, April 1996.

[55] S. Jacobsen, E. Iversen, D. Knutti, R. Johnson, and K. Biggers, "Design of the Utah/MIT dextrous hand," in Proceedings of the IEEE International Conference on Robotics and Automation, pp. 1520-1532, April 1986. 
[56] F. Lotti, P. Tiezzi, G. Vassura, L. Biagiotti, and C. Melchiorri, "UBH 3: an anthropomorphic hand with simplified endoskeletal structure and soft continuous fingerpads," in Proceedings of the IEEE International Conference on Robotics and Automation (ICRA '04), pp. 4736-4741, May 2004.

[57] J. Butterfaß, M. Grebenstein, H. Liu, and G. Hirzinger, "DLRHand II: next generation of a dextrous robot hand," in Proceedings of the IEEE International Conference on Robotics and Automation (ICRA '01), pp. 109-114, 2001.

[58] A. Kargov, T. Asfour, C. Pylatiuk et al., "Development of an anthropomorphic hand for a mobile assistive robot," in Proceedings of the IEEE 9th International Conference on Rehabilitation Robotics (ICORR '05), pp. 182-186, Chicago, Ill, USA, July 2005.

[59] C. Jones, G. Ross, J. R. Hewit, and A. P. Slade, "Robotic sorting of paper items from a random pile," Mechatronics, vol. 10, no. 8, pp. 869-880, 2000.

[60] Y. Ma, Z. Cao, X. Dong, C. Zhou, and M. Tan, "A multirobot coordinated hunting strategy with dynamic alliance," in Proceedings of the Chinese Control and Decision Conference (CCDC '09), pp. 2338-2342, June 2009.

[61] A. Ryberg, M. Ericsson, A.-K. Christiansson, K. Eriksson, J. Nilsson, and M. Larsson, "Stereo vision for path correction in off-line programmed robot welding," in Proceedings of the IEEE International Conference on Industrial Technology (ICIT '10), pp. 1700-1705, March 2010.

[62] M. Ali, R. Puffer, and H. Roman, "Evaluation of a multifingered robot hand for nuclear power plant operations and maintenance tasks," Tech. Rep., Society of Manufacturing Engineers, 1994.

[63] J. M. Romano, K. Hsiao, G. Niemeyer, S. Chitta, and K. J. Kuchenbecker, "Human-inspired robotic grasp control with tactile sensing," IEEE Transactions on Robotics, vol. 27, no. 6, pp. 1067-1079, 2011.

[64] H. Yussof, J. Wada, and M. Ohka, "Sensorization of robotic hand using optical three-axis tactile sensor: evaluation with grasping and twisting motions," Journal of Computer Science, vol. 6, no. 8, pp. 955-962, 2010.

[65] M. T. Mason and J. K. Salisbury Jr., Robot Hands and the Mechanics of Manipulation, 1985.

[66] M. T. Ciocarlie and P. K. Allen, "Hand posture subspaces for dexterous robotic grasping," International Journal of Robotics Research, vol. 28, no. 7, pp. 851-867, 2009.

[67] R. S. Dahiya, G. Metta, M. Valle, and G. Sandini, "Tactile sensing-from humans to humanoids," IEEE Transactions on Robotics, vol. 26, no. 1, pp. 1-20, 2010.

[68] K. Kim, K. R. Lee, Y. K. Kim et al., "3-Axes flexible tactile sensor fabricated by SI micromachining and packaging technology," in Proceedings of the 19th IEEE International Conference on Micro Electro Mechanical Systems (MEMS '06), pp. 678-681, Istanbul, Turky, January 2006.

[69] T.-R. Hsu, MEMS \& Microsystems: Design, Manufacture, and Nanoscale Engineering, John Wiley \& Sons, New York, NY, USA, 2008.

[70] M. Monta, N. Kondo, and Y. Shibano, "Agricultural robot in grape production system," in Proceedings of the IEEE International Conference on Robotics and Automation, pp. 2504-2509, May 1995.

[71] H. Kiba, Y. Itoh, and K. Kiryu, "Vehicle body painting robot," Google Patents, 1985.

[72] H.-S. Kang, J. Suh, and S. J. Kwak, "Welding on the fly by using laser scanner and robot," in Proceedings of the 11th International Conference on Control, Automation and Systems (ICCAS '11), pp. 1688-1691, October 2011.
[73] H.-S. Kang, J. Suh, and T.-D. Cho, "Study on robot application technology for laser welding," in Proceedings of the International Conference on Smart Manufacturing Application (ICSMA '08), pp. 354-356, Gyeonggi-do, Republic of Korea, April 2008.

[74] J. G. Webster, Tactile Sensors for Robotics and Medicine, John Wiley \& Sons, New York, NY, USA, 1988.

[75] R. H. Cannon Jr. and E. Schmitz, "Initial experiments on the end-point control of a flexible one-link robot," The International Journal of Robotics Research, vol. 3, no. 3, pp. 62-75, 1984.

[76] G. G. Hastings and W. J. Book, "Verification of a linear dynamic model for flexible robotic manipulators," in Proceedings of the IEEE International Conference on Robotics and Automation, pp. 1024-1029, 1986.

[77] A. Bernardino, M. Henriques, N. Hendrich, and J. Zhang, "Precision grasp synergies for dexterous robotic hands," in Proceedings of the IEEE International Conference on Robotics and Biomimetics (ROBIO '13), pp. 62-67, December 2013. 

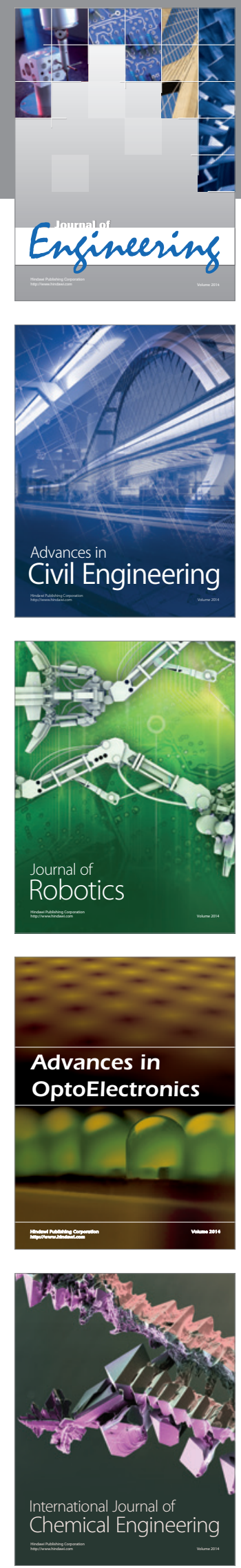

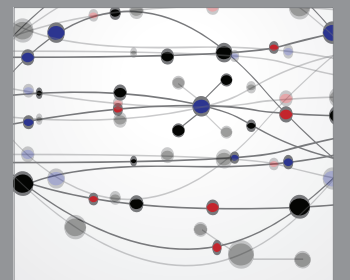

The Scientific World Journal
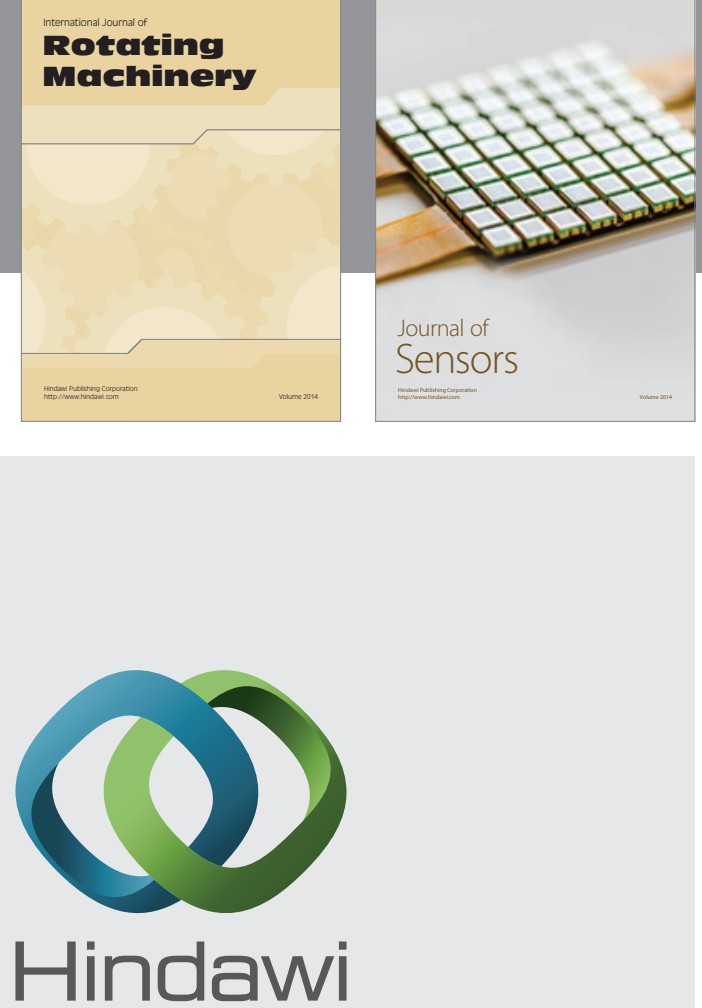

Submit your manuscripts at http://www.hindawi.com
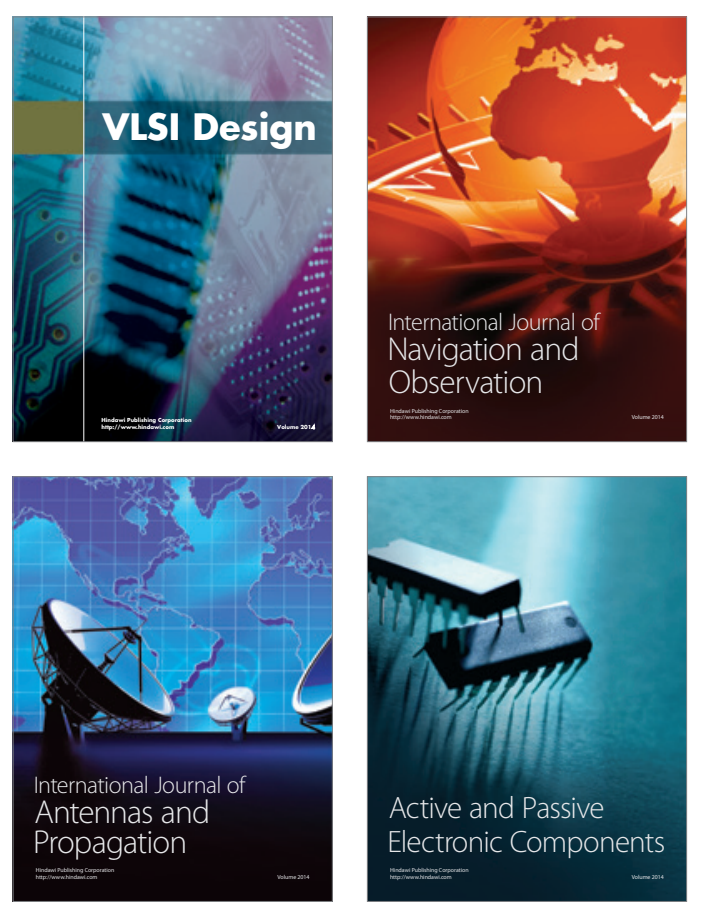
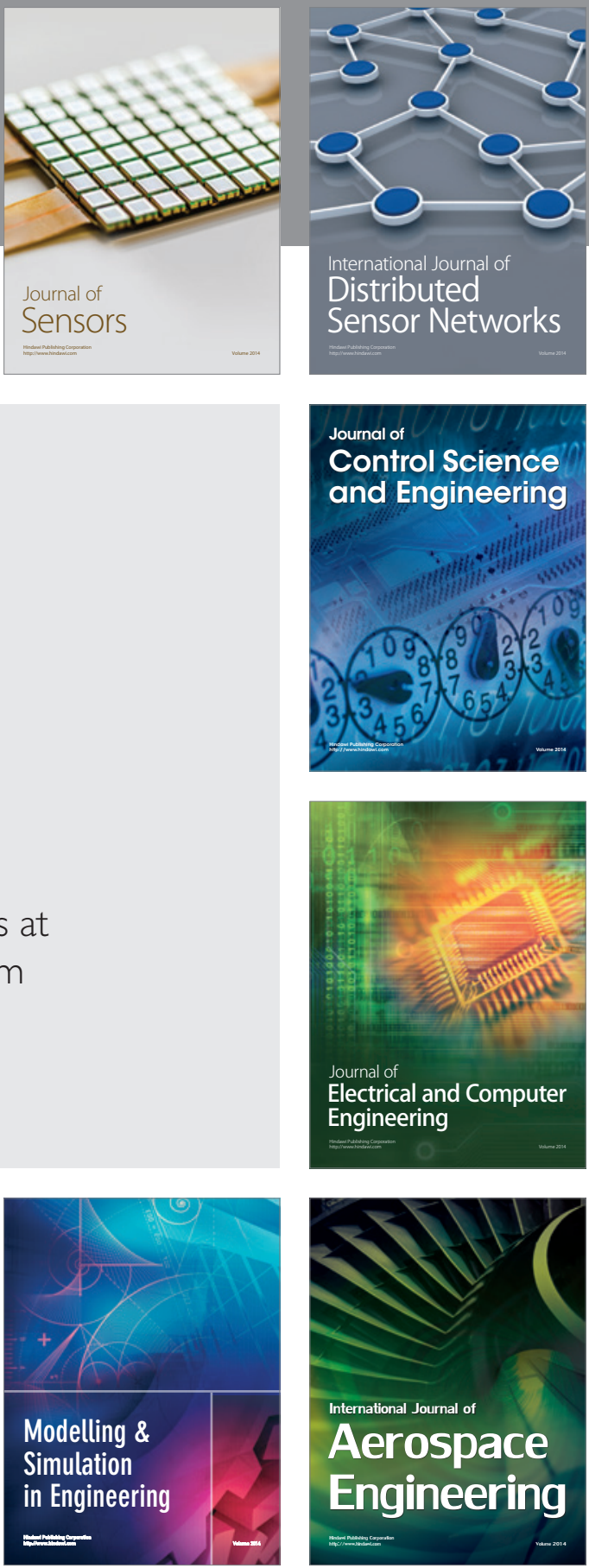

Journal of

Control Science

and Engineering
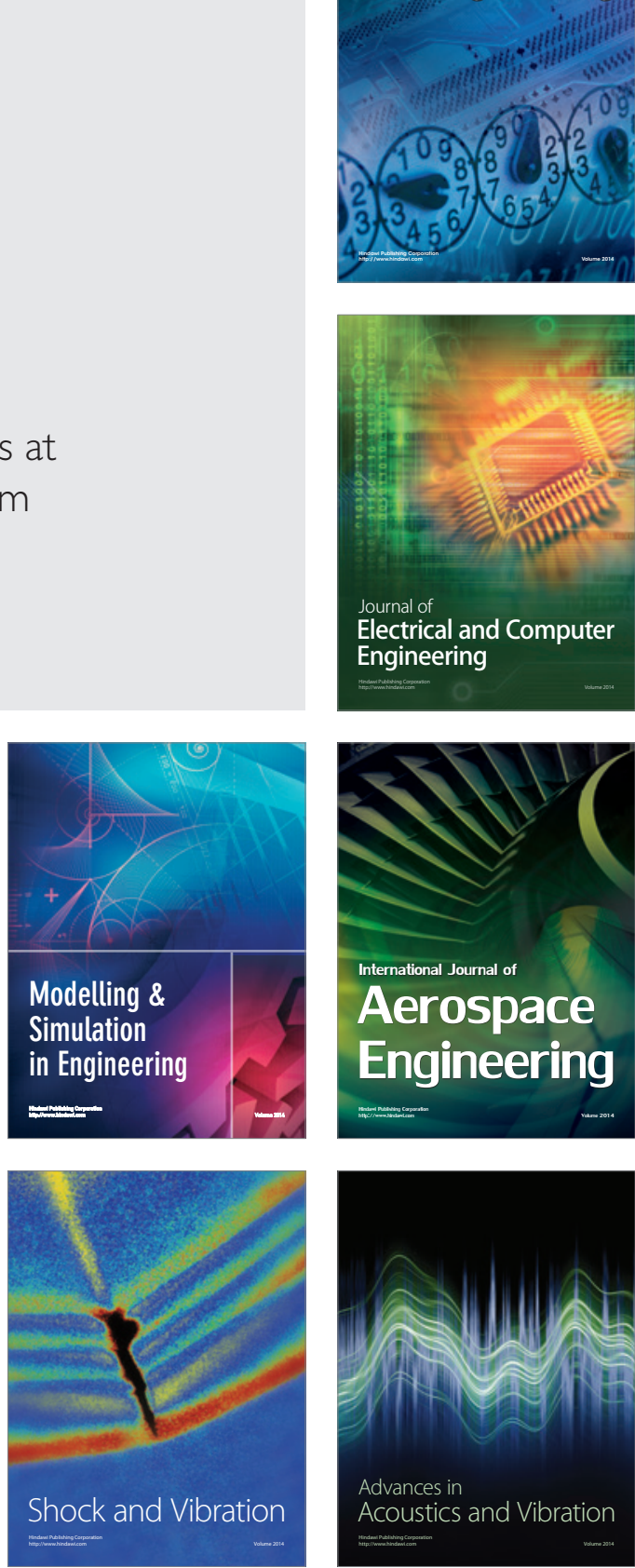